\title{
QUALIDADE E COMPOSIÇÃO QUÍMICA DE CORTES COMERCIAIS DE CARNE DE JACARÉ-DO-PANTANAL (Caiman yacare)
}

\author{
Quality and chemistry composition of comercial cuts of alligator \\ swanpland meat (Cayman yacare)
}

\author{
Erika Cristina Rodrigues ${ }^{1}$, Maria Cristina Bressan², João Vicente Neto ${ }^{3}$, Josye Oliveira e Vieira ${ }^{4}$, \\ Peter Bitencourt Faria ${ }^{5}$, Sibelli Passini Barbosa Ferrão $0^{6}$, Patricia Lopes Andrade ${ }^{7}$
}

\begin{abstract}
RESUMO
Com o objetivo de caracterizar a carne de jacaré-do-pantanal, 20 peças (cortes comerciais) foram obtidas de cinco carcaças de animais oriundos de zoocriadouros (Cáceres - MT), abatidos com peso entre 2,5 a 3,0 kg. Os cortes comparados foram: filé de cauda, filé de dorso, filé de lombo e membros. As características físico-químicas de perda de peso por cozimento (médias com variação de 38,99 a 42,28 \%); força de cisalhamento (2,29 a 2,50 kgf) e o componente de cor luminosidade $(54,01$ a 56,02$)$ foram semelhantes nos diferentes cortes. $\mathrm{O}$ teor de vermelho foi mais elevado $(\mathrm{P}<0,05)$ em membros $(2,38)$, do que em filé de lombo, de cauda e de dorso (-0,54; - 0,53 e 1,92, respectivamente). Na composição química, não houve diferença entre cortes para a proteína. Os valores foram diferentes em relação a umidade, gordura e cinzas. O corte filé de cauda mostrou $(\mathrm{P}<0,05)$ maior percentual de gordura $(0,54 \%)$, do que os cortes: filé de lombo, filé de dorso e membros $(0,29 ; 0,40$ e $0,34 \%$, respectivamente). A carne dessa espécie apresenta cortes com aparência muito clara, semelhante a coloração de pescado e quando cozidos, apresentam maciez elevada. Embora os cortes apresentem reduzidos percentuais de lipídios totais, o corte filé de cauda tem mais gordura do que os demais.
\end{abstract}

Termos para indexação: Crocodillus, Caiman yacare, qualidade de carne, composição.

\section{ABSTRACT}

With the objective of characterize the alligator swampland meat, a total of 20 parts (commercial cuts) were taken of 5 carcass from these animals kept in captivity (Cáceres MT) slaughtered with the weight ranged 2.5 to $3 \mathrm{Kg}$. The commercial cuts were tail filet, back filet, loin filet and members. The physical chemistry characterizes of weight loss (ranged means from 38.99 to $42.28 \%$ ) shear force (2.29 to $2.50 \mathrm{kgf}$ ) and the color component luminosity (54.01 to 56.02) were similar in the different cuts. However, the tenor of red was higher $(\mathrm{P}<0.05)$ for members $(2.38)$, compared with loin filet, tail filet and back filet $(0.29,0.40$ and $0.34 \%$ respectively). In the chemistry composition, any difference $(\mathrm{P}>0,05)$ among the cuts to total protein content. Therefore the results shows different in moisture, fat and ashes. The cut tail filet showed $(\mathrm{P}>0,05)$ higher fat percentage $(0,54 \%)$ than the others cuts loin filet, back filet and members $(0,29 ; 0,40$, and $0,34 \%$ respectively) These results show that the meat of alligator presents cuts with appearance very clear, similar to the color of fish, and when cooked, presents high tenderness. Also the cuts shows low percentage of total lipids and the cut tail filet show to have more fat than the others cuts.

Index terms: Crocodillus, Cayman yacare, meat quality, composition.

\section{(Recebido em 19 de abril de 2005 e aprovado em 23 de janeiro de 2006)}

\section{INTRODUÇÃO}

A utilização sustentada da vida silvestre é considerada como ação estratégica para a conservação de ambientes naturais tropicais e, a manutenção da biodiversidade. No Pantanal, devido à imprevisibilidade ambiental (secas e inundações), a carência de infraestrutura, (energia, estradas, recursos humanos e capitais), os sistemas produtivos extensivos e/ou semi-intensivos são os mais recomendados para a exploração comercial e existe mercado potencial no Brasil e no exterior para produtos diversificados no agronegócio que envolva a fauna autóctone (fauna característica de região limitada). O uso sustentado da vida silvestre é reconhecido como uma ferramenta para promover a conservação dos

\footnotetext{
${ }^{1}$ Licenciatura em Ciências Biológicas - Departamento de Ciência dos Alimentos/DCA - Universidade Federal de Lavras/UFLA - Cx. P. 3037 - $37200-000$ Lavras, MG - grikabio@yahoo.com.br

${ }^{2}$ Professora Doutora, do Departamento de Ciência dos Alimentos/DCA - Universidade Federal de Lavras/UFLA - Cx. P. 3037 - $37200-000$ - Lavras, MG bressan@ufla.br

${ }^{3}$ Mestre - Licenciatura Plena Em Ciências Agrícolas - Escola Agrotécnica Federal de Cáceres - Av. dos Ramires s/n - Cx.P. 244 - Distrito Industrial - MT - 78200-000 - eafc@terra.com.br

${ }^{4}$ Mestre. Medicina Veterinária - Total Alimentos S/A - Rodovia Fernão Dias, Km 699 - Distrito Industrial - 37410-000 - Três Corações, MG josye@totalalimentos.com.br

5Mestre. Medicina Veterinária - Escola Agrotécnica Federal de Cuiabá - Rodovia 364 Km 329 -78106-000 - Santo António do Leverger, MT _ cefet-cuiaba@cefet-cuiaba.edu.br

${ }^{6}$ Doutora. Medicina Veterinária - Universidade Estadual do Sudoeste da Bahia - Escola de Zootecnia de Itapetinga, n. 40 - $45700-000$ - Itapetinga, BA sibpass@yahoocom.br

${ }^{7}$ Medicina veterinária - Universidade Federal de Lavras/DCA - 37200-000 - Lavras, MG -patmvet@hotmail.com
} 
ambientes naturais e preservação da biodiversidade. Esse procedimento de desenvolvimento com o uso dos recursos faunísticos tem sido direcionado aos países tropicais, onde os ecossistemas naturais não foram modificados ou destruídos (COUTINHO et al., 1997).

As primeiras informações estatísticas sobre o uso econômico da fauna silvestre no Brasil apareceram nos anuários estatísticos do IBGE, a partir de 1956. Desta data até 1969, o Brasil exportou 17,9 mil toneladas de peles de animais silvestres, sendo a espécie jacaré a que produziu mais riqueza. Atualmente, a proteção e o manejo ordenado do jacaré pode trazer resultados benéficos para a região pantaneira, pois o Pantanal apresenta superpopulação desse animal e a cadeia de alimentar está fragilizada, pois o animal é voraz e ataca, principalmente, os peixes (MOURÃO, 2004).

O jacaré-do-pantanal tem seu habitat natural nas bordas da Bacia Amazônica e Bacia do Rio Paraguai (BRAZAITIS et al., 1990). É essencialmente carnívoro e a dieta varia com a idade, habitat, estação e região geográfica (WEBB et al., 1982). A dieta do jacaré, na estação da seca é constituída por insetos e peixes (SANTOS et al., 1996). Entretanto, esses consomem uma variedade de presas, incluindo crustáceos, moluscos, e vertebrados (POOLEY, 1989). Em cativeiro, é alimentado com ração em que a proteína animal é oriunda de vísceras bovinas moídas (pulmão e baço), farinha de sangue e farinha de carne (MACIEL, 2001). As normas específicas para a produção de jacaré-do-pantanal encontram-se na Portaria 126 de 13/ 02/1990 do IBAMA.

A carne, definida como tecidos animais utilizados como alimento, após a maturação e resfriamento, pode ser classificada em carnes "vermelhas" e "brancas". Dentre carnes vermelhas, as mais consumidas são oriundas de bovinos, suínos, ovinos e caprinos. As brancas são provenientes de frangos e pescados (FORREST et al., 1979).

Em estudos de análise sensorial, Romanelli (1995) destacou sua aparência atraente e o sabor agradável. Estudos recentes (HOFFMANN \& ROMANELLI, 1998) mostraram que a carne do jacarédo-pantanal tem boa aceitação, e as avaliações das propriedades demonstram potencial tecnológico para a elaboração de derivados.

O consumo da carne de animais silvestres tem aumentado e existe demanda para a exportação. Em animais silvestres, como a capivara, existem estudos avaliando: rendimento de carcaças e cortes comercias (ALBUQUERQUE, 1993; BRESSAN et al., 2002; MIGUEL,
2002), composição centesimal e colesterol de cortes comerciais (JARDIM, 2001; MIGUEL, 2002; ODA et al., 2004), características físico-químicos (BRESSAN et al., 2004; MIGUEL, 2002; ODA et al., 2004); composição de ácidos graxos (BRESSAN et al., 2004; ODA et al., 2004). Em carne de jacaré, existem estudos sobre processamento e propriedades tecnológicas (ROMANELLI et al., 2002; TELIS et al., 2003), propriedades físico-químicos (TABOGA et al., 2003; VICENTE NETO, 2005). Entretanto, em cortes comerciais foram realizadas poucas determinações. Assim, existe carência de informações que inviabilize a comercialização sistematizada, pois não é possível atender as normas brasileiras de rotulagem.

Conduziu-se este trabalho com o objetivo de determinar a composição centesimal e físico-química de cortes comerciais da carne de jacaré-do-pantanal.

\section{MATERIAL E MÉTODOS}

Empregou-se 20 cortes de carne de jacaré, oriundos de cinco carcaças de jacarés-do-pantanal (Caiman yacare), pesando entre 2,5 a $3 \mathrm{Kg}$, provenientes de zoocriadouros, autorizados pelo IBAMA, localizado no município de Cáceres - MT. Os jacarés, no pré-abate, foram submetidos a jejum de $48 \mathrm{~h}$ e permaneceram em tanques de água clorada em galpões cobertos, onde foram inspecionados $30 \mathrm{~min}$ antes do abate, lavados com água clorada e encaminhados à planta frigorífica.

Os jacarés, para as operações de abate, foram insensibilizados com pistola pneumática lançada na região do osso frontal, sangrados e desmedulizados (operação empregada após a sangria com objetivo de cessar os movimentos espasmódicos, que podem permanecer por até 40 min após a sangria, dificultando a esfola). Após, os animais esfolados foram eviscerados e resfriados à temperatura de 2 a $4{ }^{\circ} \mathrm{C}$. As carcaças, às 24 horas post mortem, foram desossadas e realizados os cortes comerciais: filé de dorso; filé de cauda; filé de lombo e membros (patas). Os cortes foram identificados, embalados em filme e congelados em "freezer" convencional (Figura 1).

As amostras, para a determinação da composição centesimal, foram homogeneizadas em multiprocessador até a obtenção de massa homogênea. A proteína foi quantificada pelo método de Kjeldahl, os lipídeos foram extraídos pelo método Soxhlet, a umidade em estufa a 105 ${ }^{\circ} \mathrm{C}$ até a obtenção de peso constante, e as cinzas em mufla a $550{ }^{\circ} \mathrm{C}$ (AOAC, 1990). As análises foram realizadas em triplicata. 


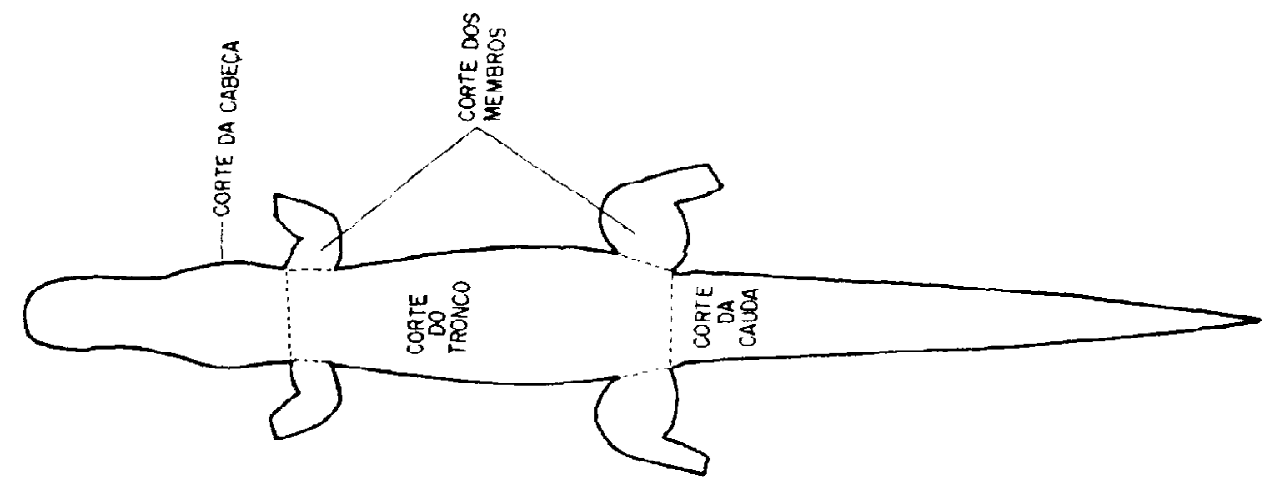

FIGURA 1 - Cortes comerciais de jacaré - figura adaptada de Romanelli (1995).

As amostras, para determinar cor, foram descongeladas em geladeira convencional. Nos cortes foram realizadas secções transversais de forma a obter três fatias de pesos semelhantes ( $1 \mathrm{~cm}$ de espessura) de cada corte e, expostos ao ambiente por 30 minutos. Após esse período, foram realizadas as leituras de cor, com colorímetro Minolta Chroma Meter, calibrado para padrão branco em ladrilho. O sistema utilizado foi o CIE L*a*b*, em que o $\mathrm{L}^{*}$ corresponde ao teor de luminosidade, $\mathrm{a}^{*}$ ao teor de vermelho e b* ao teor de amarelo (BRESSAN, 1998). Em cada fatia do corte foram realizadas quatro leituras em pontos diferentes, sendo as médias utilizadas nas análises estatísticas.

As fatias usadas para determinar cor foram usadas para avaliar a perda de peso por cozimento. Essas fatias foram identificadas, pesadas em balança semi-analítica, embaladas em papel alumínio e colocadas em chapa préaquecida a $150{ }^{\circ} \mathrm{C}$. Utilizando-se termômetro digital, a temperatura interna de cada amostra foi monitorada até atingir temperatura entre 72 e $75^{\circ} \mathrm{C}$. Ao esfriarem, em temperatura ambiente, as amostras foram pesadas e, pela diferença entre pesos inicial e final, foi calculada a perda de peso por cozimento (FELÍCIO, 1999). Na análise estatística foi utilizado a média de perda na cocção das três fatias.

As fatias cozidas usadas na PPC foram usadas para medir a força de cisalhamento (FC). De cada fatia foram retirados dois cubos de $1 \mathrm{~cm}$ de espessura (seis cubos por corte), no sentido paralelo às fibras musculares. A FC foi medida com texturômetro (m TAXT2), acoplado em célula de Warner-Bratzler, numa escala de 0 a 10. A média da FC de cada corte foi utilizada na análise estatística (os cortes filé de lombo e membros não foram avaliados, pois o tamanho desses cortes não permitiu a obtenção de número de cubos representativos da amostra).
O delineamento experimental foi em blocos casualizados para as análises de umidade, lipídeos totais, proteína, cinzas, FC, PPC e cor. O programa estatístico utilizado foi o Sisvar (FERREIRA, 2000) e o modelo experimental foi:

$$
Y i j=+C i+A j+e i j
$$

em que:

Yij = observação do corte comercial i, no animal j;

$\mathrm{m}=$ é a constante associada a todas as observações;

$\mathrm{Ci}=$ efeito dos cortes comerciais, sendo $\mathrm{i}=1,2,3$ e 4;

$\mathrm{Aj}=$ efeito dos blocos, $\mathrm{j}=1,2,3$ e 4;

eij = é o erro experimental associado à observação Yij, que, por hipótese, tem distribuição normal com média zero e variância $\sigma^{2}$.

Quando a análise de variância determinou diferença significativa, os dados foram submetidos ao teste de Tukey com nível de significância de 5\%.

\section{RESULTADOS E DISCUSSÃO}

As médias dos componentes de cor $\left(\mathrm{L}^{*} \mathrm{a} \mathrm{b}^{*}\right)$, força de cisalhamento, perda de peso por cozimento e componentes químicos (umidade, proteína, gordura e cinzas) nos diferentes cortes de jacaré são mostrados na Tabela 1.

Os diferentes cortes comerciais mostraram resultados semelhantes para o componente de cor $\mathrm{L}^{*}$, força de cisalhamento e perda de peso por cozimento. Os dados médios revelam que os cortes apresentam índices semelhantes de maciez, perda no cozimento e luminosidade.

Os resultados de PPC (sem diferença entre cortes) mostraram comportamento semelhante ao relatado por 
Vicente Neto (2005), quando esse autor comparou os cortes cauda e dorso de jacaré-do-pantanal. As médias de PPC, no presente trabalho, em cortes cujas fatias foram cozidos em grelha, as perdas foram de 38,99 a 42,28\%. Utilizando a mesma técnica, Vicente Neto (2005), em jacarés com peso entre 5 a $6 \mathrm{~kg}$ encontrou perdas inferiores $(33,55$ e 34,23 $\%)$. A diferença de resultados entre os dados atuais e os obtidos por Vicente Neto (2005) podem ser atribuídos ao tamanho da amostra (fatias). Em tilápias (Oreochromis niloticus), Ferreira (2005), utilizando métodos de cozimento, encontrou perdas de 24,69 a 45,74\% entretanto, Forrest et al. (1979) encontraram perdas no cozimento com variações de 20 a $40 \%$ e as considera normais.

As médias de FC, semelhantes nos diferentes cortes, foram de 2,29 e 2,50 kg/f para filé de cauda e filé de dorso, respectivamente. Esse comportamento de resultado foi observado em carne de capivara por Saldanha (2000) nos cortes pernil e paleta (4,70 e 4,30 kgf, respectivamente). Em jacarés de 5 a $6 \mathrm{~kg}$, Vicente Neto (2005) encontrou diferença entre os cortes cauda e dorso (3,92 e 5,20 kgf, respectivamente), que atribuiu a possível diferenciação entre o conjunto de músculos, resultado da adaptação do corpo do animal em função das diferentes atividades e demandas na natureza, conforme observado por Norkus et al. (2001), quando compararam peito e coxa de frangos (2,2 e 3,4 kgf). Com relação a valores de FC e classificações de maciez, Abularach et al. (1998) descreveram que amostras com FC até $5 \mathrm{kgf}$ podem ser consideradas maciez, mas Bickerstaffe et al. (1997) estabeleceram que carnes com até 8,00 kgf são consideradas macias, FC de 8 a 11 kgf aceitáveis e, com 11 kgf, duras. Assim, usando esses critérios, a carne de jacaré-do-pantanal pode ser considerada macia.

As médias de $\mathrm{L}^{*}$, com variação de 54,01 a 56,02. Resultados semelhantes foram relatados por Vicente Neto (2005) que encontrou médias para cauda e dorso de 57,53 e 55,28, respectivamente. Nas espécies de açougue: em bovinos são relatados médias 23,98 a 34,88 (MOONEY et al., 1998; PICALLO et al., 1998); em ovinos médias de 31,36 a 43,00 (PRADO, 2000; SOUZA, 2001); suínos médias de 49,05 a 50,21 (SILVEIRA, 1997); em peito de frango, 46,4 a 49,07 (BRESSAN, 1998; CONTRERAS, 1995). Assim, considerando esses resultados, é possível classificar a carne de jacaré como carne de luminosidade elevada (próxima às médias encontradas em carnes brancas).

Os membros $(2,38)$ e filé de dorso $(1,92)$ apresentaram médias de $\mathrm{a}^{*}$ mais elevadas $(\mathrm{P}<0,05)$, do que os cortes filé de lombo e filé de cauda (-0,54 e -0,53, respectivamente). Comportamento semelhante foi observado por Francesco et al. (2004), que trabalhando com trutas (Oncorhynchus mykiss) e comparando porções da carcaça (dorso, ventre e cauda), observaram diferenças entre porções musculares para os componentes de cor. Essa diferença pode ser

TABELA 1 - Médias dos componentes de cor (L*a*b*), FC, PPC, e composição centesimal em carne de jacaré-dopantanal.

\begin{tabular}{|c|c|c|c|c|}
\hline Determinações & Filé de lombo & Filé de dorso & Filé cauda & Membros \\
\hline \multicolumn{5}{|l|}{ Cor } \\
\hline $\mathrm{L}^{*}$ & $54,01^{\mathrm{a}}$ & $54,02^{\mathrm{a}}$ & $55,48^{\mathrm{a}}$ & $56,02^{\mathrm{a}}$ \\
\hline$a^{*}$ & $-0,54^{\mathrm{b}}$ & $1,92^{\mathrm{a}}$ & $-0,53^{\mathrm{b}}$ & $2,38^{\mathrm{a}}$ \\
\hline$b^{*}$ & $-1,50^{\mathrm{b}}$ & $-0,79^{b}$ & $-2,61^{b}$ & $1,77^{\mathrm{a}}$ \\
\hline $\mathrm{FC}$ & - & $2,50^{\mathrm{a}}$ & $2,29^{\mathrm{a}}$ & - \\
\hline PPC & $38,99^{\mathrm{a}}$ & $39,71^{\mathrm{a}}$ & $40,34^{\mathrm{a}}$ & $42,28^{\mathrm{a}}$ \\
\hline Umidade & $76,68^{\mathrm{ab}}$ & $77,18^{\mathrm{a}}$ & $76,75^{\mathrm{ab}}$ & $75,44^{\mathrm{b}}$ \\
\hline Proteína & $24,23^{\mathrm{a}}$ & $24,37^{\mathrm{a}}$ & $23,57^{\mathrm{a}}$ & $24,10^{\mathrm{a}}$ \\
\hline Gordura & $0,29^{\mathrm{a}}$ & $0,40^{\mathrm{ab}}$ & $0,54^{\mathrm{b}}$ & $0,34^{\mathrm{ab}}$ \\
\hline Cinzas & $0,99^{\mathrm{b}}$ & $0,82^{\mathrm{ab}}$ & $0,58^{\mathrm{a}}$ & $0,82^{\mathrm{ab}}$ \\
\hline
\end{tabular}

Médias seguidas por mesma letra nas colunas, não diferem entre si pelo teste de Tukey $(\mathrm{P}<0,05)$. 
atribuída as diferentes disposições musculares, tamanhos e tipos de fibras e retenção de pigmentos carotenóides (FORREST et al., 1979). Entretanto, Vicente Neto (2005) não encontrou diferença entre cauda e dorso $(-0,24$ a 0,07), respectivamente.

Comparando o teor de vermelho nas diferentes espécies: em bovinos são relatadas médias de 12,28 a 14,64 (MONEY et al., 1998; PICALLO et al., 1998); em ovinos, médias de 10 a 18,01 (PRADO, 2000; SOUZA, 2001); em suínos, de 5,50 a 5,94 (SILVEIRA, 1997); em frangos, 1,90 a 3,83 (BRESSAN, 1998; CONTRERAS, 1995; NORKUS et al., 2001). Assim, considerando esses dados é possível classificar a carne de jacaré como carne com baixo teor de vermelho.

A média de $b^{*}$ para membro $(1,77)$ foi superior $(\mathrm{p}<0,05)$ em relação às médias dos demais cortes (filé de cauda; filé de dorso e filé de lombo com -2,61; -0,79 e 1,50 , respectivamente). Porções musculares com diferentes médias foram relatadas por Francesco et al. (2004), em truta nos diferentes cortes: dorso (12,91 a 15,02); ventre (10,53 a 12,34) e cauda (12,35 a 13,59) e por Norkus et al. (2001), em peito e coxa de frango (5,39 a 6,94, respectivamente). Entretanto, Vicente Neto (2005) não encontrou diferenças para o teor de amarelo entre os cortes cauda e dorso $(-0,13$ e $-0,30$, respectivamente).

Os teores de amarelo encontrados na literatura para outras espécies como bovino são de 7,29 a 9,08 (MONEY et al., 1998; PICALLO et al., 1998), ovinos de 3,34 a 8,15 (PRADO, 2000; SOUZA, 2001); suínos de 5,80 a 6,53 (SILVEIRA, 1997); frangos de 4,1 a 5,6 (CONTRERAS, 1995). Assim, comparando as diferentes médias dos animais domésticos é possível definir a carne de jacaré como carne com baixo teor de amarelo.

Os cortes apresentaram diferenças $(\mathrm{P}<0,05)$ umidade, gordura e cinza. Entretanto não houve diferença para proteína. O filé de dorso mostrou média mais elevada $(77,18 \%)$ de umidade $(\mathrm{P}<0,05)$, umidade semelhante nos cortes filés de cauda e filé de lombo (76,75 e 76,68\%, respectivamente) e menor porcentagem foi encontrada nos membros $(75,44 \%)$. Em jacaré, não relataram diferenças: Vicente Neto (2005) entre cauda e dorso (73,39 e 76,45\%, respectivamente), e Moody et al. (1980), em cortes de jacaré (Alligator mississippiensis), cujas médias variaram 73 a 76,8 \%. Embora não tenha comparado cortes, Romanelli (1995) obteve médias de 78,37 a 75,23\% para animais de 2 a $4 \mathrm{~kg}$ e de 16,5 a $20,9 \mathrm{~kg}$.

Não houve diferença entre os cortes para as médias de proteína (23,57 a 24,37 \%). Resultado semelhante foi reportado por Vicente Neto (2005) para cauda e dorso que citou média de 23,01 e $22,80 \%$, respectivamente, e por Moody et al. (1980) que reportaram valores de 21,1 a 22,3 $\%$ em jacaré americano em quatro cortes. As médias, no presente trabalho, foram superiores aos valores relatados para espécies domésticas com médias variando de 17,54 a 20,4 \% (NORKUS et al., 2001; PALEARI et al., 1998; SOUZA, 2001) em bovinos, ovinos, perus e frangos. Embora, Romanelli (1995) tenha citado baixos valores de proteínas (18,40 a 18,43\%) em longissimus de jacaré-do-pantanal.

As médias de extrato etéreo (EE) foram superiores $(\mathrm{P}<0,05)$ para filé de cauda $(0,54 \%)$, semelhante para os cortes filé de dorso e filé de membros $(0,40$ e $0,34 \%$, respectivamente) e menor EE foi encontrado no filé de lombo $(0,29 \%)$. Vicente Neto (2005), comparando cortes, também encontrou quantidade de EE mais elevado no corte cauda $(3,13 \%)$, do que no corte dorso $(0,51 \%)$. A diferença entre os resultados do presente trabalho e os dados de Vicente Neto (2005) é decorrente dos diferentes pesos dos jacarés. Em animais domésticos (mamíferos) durante o crescimento, a umidade do tecido muscular vai sendo substituída pelo tecido adiposo, e essas alterações são mais intensas na fase de terminação (NICKELL \& BROMAGE, 1998; SOUZA et al., 2002). Nas espécies silvestres, os lipídios representam menos de $5 \%$ do peso da carcaça (gordura estrutural ou na forma de fosfolipídios), e em animais domésticos, a gordura é maior que $20 \%$ do peso da carcaça.

$\mathrm{O}$ teor de cinzas mostrou-se elevado $(\mathrm{P}<0,05)$ no corte filé de lombo com média de $0,99 \%$, seguido dos cortes filé de dorso $(0,82 \%)$, membros $(0,82 \%)$ e filé da cauda $(0,58$ $\%)$. Vicente Neto (2005) também encontrou diferença entre cauda e dorso com médias de 1,00 e 1,12\%, respectivamente, e Romanelli (1995) relatou média de 1,02\% para cinzas.

\section{CONCLUSÕES}

O corte filé de cauda apresenta porcentagem mais elevada de gordura e mais baixo de cinzas quando comparado aos outros cortes de jacaré do Pantanal. Não há diferença entre os cortes para os percentuais de proteína, cinzas e colesterol. O corte filé de cauda apresenta menor porcentagen em umidade e proteína.

\section{AGRADECIMENTOS}

Os autores agradecem ao CNPQ (1 bolsa produtividade, 1 bolsa de mestrado da aluna Josye Oliveira e Vieira), a CAPES (1 bolsa de doutoramento da aluna Sibelli Passini Barbosa Ferrão - PICDT; 1 bolsa de mestrado do aluno Peter Bitencourt Faria); e a Cooperativa de produtores de Jacaré-do-Pantanal (COOCRIJAPAN), pela doação das carcaças dos animais. 


\section{REFERÊNCIAS BIBLIOGRÁFICAS}

ABULARACH, M. L. S.; ROCHA, C. E.; FELÍCIO, P. E. Características de qualidade de contra filé ( m.l. dorsi) de touros jovens da raça nelore. Ciência Tecnologia de Alimentos, Campinas, v. 18, n. 2, p. 205-210, 1998.

ALBUQUERQUE, N. I. Ganho de peso na fase final de crescimento e sistematização da avaliação de carcaça de três categorias de capivara (Hydrochaeris hydrochaeris): machos inteiros, machos castrados e fêmeas. 1993. $56 \mathrm{f}$. Dissertação (Mestrado) - Escola Superior de Agricultura Luiz de Queiroz, Piracicaba, 1993.

ASSOCIATION OF OFFICIAL ANALYTICAL CHEMISTS. Official methods of analysis of the Association of Official Analytical Chemists. 15. ed. Arlington, 1990.

BICKERSTAFFE, R.; COUTEUR, C. E.; MORTON, J. D. Consistency of tenderness in New Zealand retail meat 43th I CoMST. [S.1.: s.n.], 1997.

BRAZAITIS, P.; YAMASHITA, C.; REBÊLO, G. H. A summary report of the CITES central South American caiman study: phase I: Brazil. In: WORKING MEETING OF CROCODILE SPECIALIST GROUP, 9., 1990, Gland. Proceedings... Gland: The World Conservation Union, 1990. p. 100-115.

BRESSAN, M. C. Fatores dos fatores pré e pós-abate sobre a qualidade da carne de peito de frango. 1998. $201 \mathrm{f}$. Tese (Doutorado em Tecnologia de Alimentos) - Universidade Estadual de Campinas, Campinas, 1998.

BRESSAN, M. C.; MIGUEL, G. Z.; FARIA, P. B.; VIEIRA, J. O.; ODA, S. H. I. Rendimento de carcaça e de cortes comerciais de capivaras (Hydrochaeris hydrochaeris 1.1766). Ciência e Agrotecnologia, Lavras, p. 1588-1593, dez. 2002.

BRESSAN, M. C.; ODA, S. H. I.; CARDOSO, M. G.; MIGUEL, G. Z.; FREITAS, R. T. F.; VIEIRA, J. O.; FARIA, P. B.; SAVIAN, T. V.; FERRÃO, S. P. B. Composição de ácidos graxos dos cortes comerciais de capivara (Hydrochaeris hidrochaeris L.1766). Ciência Agrotecnologia, Lavras, v. 28, n. 6, p. 1352-1359, 2004.

CONTRERAS, C. J. C. Efeitos do atordoamento elétrico, estimulação elétrica $\mathrm{e}$ da desossa à quente na qualidade da carne do peito de frango "pectoralis major". 1995. 150 f. Tese (Doutorado em Tecnologia de Alimentos) Universidade Estadual de Campinas, Campinas, 1995.

COUTINHO, M.; CAMPOS, Z.; MOURÃO, G.; MAURO, E. R. Aspectos ecológicos dos vertebrados terrestres e semi-aquáticos no Pantanal. In: BRASIL. Ministério do Meio Ambiente, dos Recursos Hídricos e da Amazônia Legal. Plano de conservação da Bacia do Alto Paraguai, pantanal: diagnóstico dos meios físicos e bióticos. Brasília, DF, 1997. v. 2, p. 183-322.

FELICIO, P. E. Qualidade da carne bovina: características físicas e organolépticas. In: REUNIÃO ANUAL DA SOCIEDADE BRASILEIRA DE ZOOTECNIA, 36., 1999, Porto Alegre. Anais... Porto Alegre: SBZ, 1999. p. 89-97.

FERREIRA, D. F. Análises estatísticas por meio do Sisvar para Windows versão 4.0. In: REUNIÃO ANUAL DA REGIÃO BRASILEIRA DA SOCIEDADE INTERNACIONAL DE BIOMETRIA, 45., 2000, São Carlos, SP. Anais... São Carlos: UFSCar, 2000. p. 255-258.

FERREIRA, M. W. Composição química e perfil lipídio do filé de tilápia do nilo (Oreochromis niloticus, Linnaeus 1757) cru e submetidos a diferentes métodos de cocção. 2005. 120 p. Dissertação (Mestrado em Ciência dos Alimentos) - Universidade Federal de Lavras, Lavras, 2005.

FORREST, J. C.; ABERLE, E. D.; HEDRICK, H. B.; JUDGE, M. D.; MERKEL, R. A. Fundamentos de ciência de la carne. Traduzido por Barnabé Sans Pérez. Zaragoza: Acribia, 1979. 364 p. Tradução de: Principles of meat sciense.

FRANCESCO, M.; PARISI, G.; ME`DALE, F.; LUPI, P.; KAUSHIK, S. J.; POLI, B. M. Effect of long-term feeding with a plant protein mixture based diet on growth and body/ fillet quality traits of large rainbow trout (Oncorhynchus mykiss). Aquaculture, Amsterdam, v. 236, p. 413-429, 2004.

HOFFMANN, F. L.; ROMANELLI, P. F. Análise microbiológica da carne de jacaré do pantanal (Caiman crocodillus yacare). Ciência Tecnologia Alimentos, Campinas, v. 18, n. 3, p. 258-264, 1998.

JARDIM, N. S. Sexo e diferentes pesos ao abate na qualidade da carne de capivaras (Hydrochaeris hidrochaeris L. 1766). 2001. 119 p. Dissertação (Mestrado em Ciência dos Alimentos) - Universidade Federal de Lavras, Lavras, 2001. 
MACIEL, F. R. Coeficiente de digestibilidade aparente de cinco fontes energéticas para o jacaré-do-pantanal (Caiman yacare, Daudin, 1802). 2001. 76 p. Dissertação (Mestrado em Zootecnia) - Universidade Federal de Lavras, Lavras, 2001.

MIGUEL, G. Z. Caracterização da carcaça e da carne de capivaras (Hydrochaeris hydrochaeris L. 1766) em idade adulta. 2002. 107 p. Dissertação (Mestrado em Ciência de Alimentos) - Universidade Federal de Lavras, Lavras, 2002.

MOODY, M.; COREIL, P. D.; RUTLEDGE, J. E. Alligator meat: yields, quality studied. Lousiana Agriculture, Lousiana, v. 24, n. 1, p. 14-15, 1980.

MOONEY, M. T.; FRENCH, P.; MOLONEY, A. P.; O'RIORDAN, E.; TROY, D. J. Quality differences between hrbage and concentrate-fed beef animals. In: INTERNATIONAL CONGRESS OF MEAT SCIENCE AND TECHNOLOGY, 44., 1998, Barcelona. Anais... Barcelona: ICOMST, 1998.

MOURÃO, G. M. Utilização econômica da fauna silvestre no Brasil: o exemplo do jacaré-do-pantanal. Brasília, DF: Embrapa, 2004. Disponível em: <htitp://WwW.embrapa.br/ Acesso em: 3 jun. 2004.

NICKELL, D. C.; BROMAGE, N. R. The effect of timing duration of feeding astaxanthin on the development and variation of fillet colour and efficieney of pigmentation in rainbow trout (Oncorchynchus mykiss). Aquaculture, Amsterdam, n. 169, p. 233-246, 1998.

NORKUS, E. A.; SOUZA, H. B. A.; SOUZA, P. A.; OBA, A.; KODAWARA, L. M.; LEONEL, F. R.; PELICANO, E. R. L. Avaliação da qualidade física e química da carne de frangos abatidos em diferentes idades. In: CONGRESSO BRASILEIRO DE CIÊNCIA E TECNOLOGIA DE CARNES, 1., 2001, São Pedro, SP. Anais... São Pedro: USP, 2001. p. 203-204.

ODA, S. H. I.; BRESSAN, M. C.; FREITAS, R. T. F.; MIGUEL, G. Z.; VIEIRA, J. O.; FARIA, P. B.; SAVIAN, T. V. Composição centesimal e teor de colesterol dos cortes comerciais de capivara (Hydrochaeris hydrochaeris L. 1766). Ciência e Agrotecnologia, Lavras, v. 28, n. 6, p. 1344-1351, 2004.

PALEARI, M. A.; CAMISASCA, S.; BERETTA, G.; RENAN, P.; CORISCO, P.; BERTOLO, G.; CRIVELLI, G. Ostrich meat physico chemical characteristics and comparison with turkey and bovine meat. Meat Science, Oxford, v. 48, n. 3/ 4, p. 205-210, Mar./Apr. 1998.

PICALLO, A. B.; SANCHO, A. M.; MARGARÍA, C. A.; LASTA, J. A. Colour and tenderness relationships in different steer breeds. In: INTERNATIONAL CONGRESS OF MEAT SCIENCE AND TECHNOLOGY, 44., 1998, Barcelona. Anais... Barcelona: ICOMST, 1998.

POOLEY, A. C. Dieta y habitos alimentares. In: ROSS, C. A. (Ed.). Cocodrilos y caimanes. Barcelona: Espanha Fundácion, 1989.

PRADO, O. V. Qualidade de carne de cordeiros Santa Inês e Bergamácia abatidos em diferentes pesos. 2000. 109 p. Dissertação (Mestrado em Zootecnia) - Universidade Federal de Lavras, Lavras, 2000.

ROMANELLI, P. F Propriedades tecnológicas da carne do jacaré do pantanal Caiman Crocodilus Yacare (Daudin, 1802). 1995. 110 f. Tese (Doutorado em Tecnologia de Alimentos) - Universidade de Campinas, Campinas, 1995.

ROMANELLI, P. F.; CESARI, R.; LIMA FILHO, J. F. Processamento da carne do jacaré-do-Pantanal (Caiman crocodillus yacare). Ciência e Tecnologia de Alimentos, Campinas, v. 22, n. 1, p. 70-75, 2002.

SALDANHA, T. Determinação da composição centesimal nos diferentes cortes da carne de capivara (Hydrochoerus hydrochaeris). 2000. 105 f. Dissertação (Mestrado) Universidade Federal Rural do Rio de Janeiro, Rio de Janeiro, 2000.

SANTOS, S. A.; PINHEIRO, M. S.; SILVA, R. A. Efeitos de diferentes dietas naturais no desenvolvimento inicial de Caiman crocodillus yacare (Crocodilia alligatoridae). Revista da Sociedade Brasileira de Zootecnia, Viçosa, v. 22, p. 406-412, 1996.

SILVEIRA, E. T. F. Técnicas de abate e seus efeitos na qualidade da carne suína. 1997. 226 f. Tese (Doutorado em Tecnologia de Alimentos) - Universidade Estadual de Campinas, Campinas, 1997.

SOUZA, X. R. Efeitos de grupo genético, sexo e peso ao abate na qualidade de carne de cordeiros em crescimento. 2001. 116 p. Dissertação (Mestrado em Ciência dos Alimentos) - Universidade Federal de Lavras, Lavras, 2001. 
SOUZA, X. R.; PEREZ, J. R. O.; BRESSAN, M. C.; LEMOS, A. L. S. C.; BONAGURIO, S.; GARCIA, I. F. F. Composição centesimal do músculo biceps femoris de cordeiros em crescimento. Ciência e Tecnologia de Alimentos, Campinas, p. 1507-1513, dez. 2002. Edição especial.

TABOGA, S. R.; ROMANELLI, P. F.; FELISBINO, S. L.; BORGES, L. F. Post-mortem alterations (Glycolysis) of pantanal alligator's (Caiman crocodilus yacare) muscle. Ciência e Tecnologia de Alimentos, Campinas, v. 23, n. 1, p. 2-27, 2003.

TELIS, V. R. N.; ROMANELLI, P. F.; GABAS, A. L.; TELISROMERO, J. Salting kinetics and diffusiuities in farmed
Pantanal Caiman muscle. Pesquisa Agropecuária Brasileira, Brasília, v. 38, n. 4, p. 529-535, 2003.

VICENTE NETO, J. Caracterização físico química, colesterol e ácidos graxos da carne de jacaré-do-pantanal (Caiman yacare, Daudin 1802) oriundo de zoocriadouro e habitat natural. 2005. 156 p. Dissertação (Mestrado) Universidade Federal de Lavras, Lavras, 2005.

WEBB, G. J. W.; MANOLIS, S. C.; BUCKWORTH, R. Crocodillus johnstoni in the McKinlay River Area, N.T.I. variation in diet, and a new method of assessing the relative importance of prey. Australian Journal Zoology, Melbourne, v. 30, p. 877-899, 1982. 